DOI https://doi.org/10.30525/978-9934-26-008-7.1-3

\title{
DIAMOND SPARK GRINDING IN ANHYDRATED MEDIUM USING SOLID LUBRICANTS
}

\author{
Gutsalenko Yu. G., Rudnev A. V.
}

\section{INTRODUCTION}

The technological advantages of diamond spark grinding (DSG), emanating from the stable development of the cutting relief, are due to the action of electric discharges in the processing zone, which is included in the electrical circuit that generates them ${ }^{1,2,3}$. Ozone therapy of the environment, which is characteristic of the action of electric discharges in the air, allows us to consider this method of treatment in a number of environmentally promising by it's nature ${ }^{4}$.

The support of the development of wheel working relief by the action of electric discharges on the its bond significantly inhibits the growth of thermo-mechanical tension of mass microcutting in the zone of DSG over time. The introduction of tracking control directly over the formation of the cutting relief (its height and sharpness) or the parameters of the thermomechanics of grinding (temperature regime, grinding power, power load in the direction of the main movement to material removal and on unproductive dissipation of consumed energy), with a feedback mechanism in the control of electrical modes in the circuit of initiation of electrical discharges according to a certain algorithm, allows to maintain the thermomechanical tension of the working process at a given level, below the average indicators of conventional diamond grinding with the same level of operating performance. The reduced level of thermomechanical stress of DSG in production operations with comparable mechanics is an a priori attractive advantage of the process for testing the minimum lubrication methodology.

1 Kobzar, L. E., V. A. Fadeev, and N. K. Bezzubenko (1995) Progressive diamond-spark grinding. Kharkov State Polytech. Univ., FED Sc.-Prod. Assoc., Kharkov (in Russian).

${ }^{2}$ Uzunyan, M. D. (2003) Diamond-spark grinding of hard alloys. NTU "KhPI", Kharkov (in Russian).

3 Gutsalenko, Yu. G. (2018) Diamond-spark grinding of high functionality materials, $3^{\text {rd }} E d$. Cursor, NTU "KhPI", Kharkov (in Russian).

${ }^{4}$ Gutsalenko, Yu., and C. Iancu (2020) Fiability \& Durability, 1(25)/2020: 5-10. 


\section{Ecological relevance and technically competitive environment}

Parts with high functionality due to the increased hardness and durability of manufacturing materials, that is hard-to-process materials, are widely used in industries that determine the technological and technological competitiveness of developed countries. Simultaneously ensuring technological productivity, functional quality and environmental cleanliness of technologies in the manufacture of parts from such materials is a major complex problem.

Generally accepted technological methods for accurate finishing grinding of difficult-to-work parts consist in use of jet irrigation with lubricatingcooling technological means (LCTM) on water or oil liquid basis 5 .

In the industrialized countries of the world, a large number of liquid LCTM are used, for example in Germany and the USA up to 110 and 250 million liters per year, respectively. But the intercontinental scale of the problem of the provision of clean fresh water of vast territories in the Asian, African, Australian, American regions is globally stimulating the reduction of water consumption for industrial purposes as a permanent trend in the scientific and technological development of our civilization ${ }^{6}$, including with regard to LCTM during machining - from minimizing the flow, for example in the form of partial microdoses, and to complete rejection ${ }^{7}$.

The LCTM is a source of relatively high production costs associated with its preparation, transportation, operation of feed systems to the treatment area, regeneration, recovery and disposal. According to the Swiss firm Mikron SA Aqno ${ }^{8}$, the cost of LCTM per machine is an average of \$ 50 to \$ 300 per day, taking into account the full cost of the LCTM filing systems in the treatment area, maintaining its normal condition.

The current relevance of minimizing the consumption of water resources for industrial technologies, including water-based lubricating and cooling technological agents, is associated with multi-regional drinking water hunger as a global environmental challenge to the development of civilization ${ }^{9,10}$ and meets the modern technocratic

${ }^{5}$ Winter, M. (2016) Eco-efficiency of Grinding Processes and Systems. Springer International Publishing AG Switzerland.

${ }^{6}$ Collins, A., et al. (2019) The Global Risk Report 2019, $14^{\text {th }}$ Ed. WEF, Geneva.

${ }^{7}$ Benedicto, E., et al. (2017) Technical, economic and environmental review of the lubrication/cooling Procedia Eng. 184 99-116.

${ }^{8}$ Micron Annual Report 2018 (2018). Micron Holding AG, Biel.

${ }^{9}$ Winter, M. (2016) Eco-efficiency of Grinding Processes and Systems. Springer International Publishing AG Switzerland.

${ }^{10}$ Collins, A., et al. (2019) The Global Risk Report 2019, $14^{\text {th }}$ Ed. WEF, Geneva. 
strategies of the European Union (Industry 4.0) ${ }^{11}$, Japan (Society 5.0) ${ }^{12}$, China (Made in China 2025) ${ }^{13}$ and other leading world economies ${ }^{14}$.

The work being announced is a response to the environmental challenge, also taking into account economic considerations, through the refusal to use water resources in LCTM compositions and the proposal of innovative anhydrous processes of diamond-spark grinding (DSG) of difficult-to-process materials materials using solid lubricants (SLs).

An analysis of the activity of firms and research centers shows that there is an intense search in the world for efficient technological methods of transition to low and waterless machining technologies in line with the global environmental challenge of drinking water. The problem of cooling systems in the process of grinding materials is updated by the modern European technology platform Industry 4.0 (M. Winter, Germany ${ }^{15}$ ) and is considered in the unity of technical, economic and environmental development (E. Benedicto (Spain), D. Carou (Ireland) and M. Rubio (Portugal) ${ }^{16}$ ).

In China and the USA, ideas about the thermodynamic mechanism of grinding processes are formed under conditions of minimal cooling and lubrication (M. Yang and others ${ }^{17}$ ). Heat removal from the cutting zone using minimal liquid lubrication alternative to conventional approaches is investigating by finite element models (M. Hadad and A. Sharbati, Iran ${ }^{18}$ ). Unconventional inclusion of the grinding wheel in the flow of cooling agents in the processing of difficult-to-process materials is developing

${ }^{11}$ Smit, J., et al. (2016) Industry 4.0: Study for the European Parliament's Committee on Industry, Research and Energy. EP, Directorate General for Internal Policies, Policy Dept A: Econ. and Sci. Policy, Brussels.

${ }^{12}$ Toward realization of the new economy and society - reform of the economy and society by the deepening of "Society 5.0" (2016). Keidanren (Japan Business Federation), Tokyo.

${ }_{13}$ Made in China 2025: Global ambitions built on local protection (2017). U.S. Chamber of Commerce, Washington.

${ }^{14}$ Gutsalenko Yu., et al. (2019) Annals of the „Constantin Brancusi” University of Targu Jiu, Engineering Series, 2/2019: 13-17.

${ }^{15}$ Winter, M. (2016) Eco-efficiency of Grinding Processes and Systems. Springer International Publishing AG Switzerland.

${ }^{16}$ Benedicto, E., et al. (2017) Technical, economic and environmental review of the lubrication/cooling Procedia Eng. 184 99-116.

17 Yang, M., et al. (2018) In: Microfluidics and Nanofluidics. IntechOpen, London, 61-81.

${ }^{18}$ Hadad, M., and A. Sharbati (2016) Procedia CIRP 40 509-515. 
(S. Toyokawa and others, $\operatorname{Japan}^{19}$ ). Various solid alternatives to liquid remedies that are grease-cooling and clean the grinding wheel surface down to hard water (dry ice) are considered (Yu. Ohta and others, $\operatorname{Japan}^{20}$ ).

New opportunities for comprehensive optimization of grinding operations in countries with fresh water problems are associated with the improvement of air cooling (B. Boswell and others, Australia and Indonesia ${ }^{21}$ ). Air flow as a cooling medium when cutting materials is proposed to be pre-activated by ionization (M. Sh. Migranov and others, Russia $^{22}$ ). Regional cultures of clean water savings and the global nature of the problem also stimulate international scientific and technical cooperation in the development and research of minimal lubrication techniques as a viable alternative to conventional irrigation (B. Sen and others $^{23}$, India, Bangladesh, Poland and the United Kingdom).

Perspective approaches to the development of environmentallyfriendly productive diamond grinding processes for difficult-to-process materials include the use of anhydrous SL processing and minimal liquid cooling (M. Winter, Germany ${ }^{24}$ ), but known information on relevant research practices in the leading economically advanced research schools (USA, The United Kingdom, Germany, China, Japan) and others are, first and foremost, fragmented and not sufficient to develop technological instructions and industry recommendations. Secondly, it is possible to claim that there are no such studies regarding the most advanced electrophysical methods of diamond-abrasive processing, except for some experience of DSG at the NTU "KPI" with the study of microgeometric heredity of the process on hard alloys (A. K. Agu and M. D. Uzunyan ${ }^{25}$, Nigeria and Ukraine).

\section{The main idea, hypotheses and bases}

The central idea of the work is to use the advantages of diamond grinding with the developed cutting surface of the working surface of the

19 Toyokawa, S., et al. (2019) Key Eng. Mater. 825 84-91.

${ }^{20}$ Ohta, Yu., et al. (2017) Key Eng. Mater. 749 124-129.

${ }^{21}$ Boswell, B., et al. (2016) Lect. Notes Eng. Comput. Sci. 2 680-687.

22 Migranov, M. Sh., et al. (2018) Bulletin of the UGATU 22 (4(82)): 12-18 (In Russian).

${ }^{23}$ Sen, B., et al. (2019) Int. J. Pr. Eng. Man.-GT. In press, publ. online: 15 Oct. 2019.

${ }^{24}$ Winter, M. (2016) Eco-efficiency of Grinding Processes and Systems. Springer International Publishing AG Switzerland.

${ }^{25} \mathrm{Agu}$, A. C., and M. D. Uzunyan (2017) Cutting and tooling in technological systems 91 12-17 (In Russian). 
wheel from the standpoint of the thermal load of the cutting zone and therefore less dependence on the intensive use of LCTM, in the usual practice - jet irrigation; the possibility of transition through the application of SLs to the global trend of reducing water consumption in the industry due anhydrous diamond-abrasive processing technologies; solution on this basis of a complex problem of technological productivity, function the quality and ecological purity of diamond grinding of difficult-to-process materials.

Previous studies by the authors on the processes of diamond grinding with the initiation of electric discharges (DSG) and without electric current in the treatment $z o n e^{26,27}$ allow to assume the less significant thermo-physical consequences of the engineering of the treated surface than the more developed and stabled a cutting relief of the wheel working surface during grinding.

The consequence of this hypothesis is the expectation of a much smaller dependence of the productivity and quality of diamond grinding processes with developed wheel cutting relief from the LCTM.

From the point of view of the cleaning of the wheel working surface from the products of chip formation during processing, the important working hypothesis is the electro-physical by nature, to a large extent, the equivalence of anhydrous DSG processes to the processes of diamond grinding with the function of jet cleaning and washing of the wheel working surface from the chip products.

From the standpoint of reducing heat intensity in the treatment area, an important working hypothesis is largely tribological in nature, the equivalence of anhydrous diamond-spark grinding processes using SL to processes of diamond grinding with jet irrigation.

Among other working hypotheses, the following are more significant:

1) SLs of the work surface of a diamond grinding wheel can be considered as a coating with a high wear rate, which is renewed according to a certain technological regulation, that is, in the function of a certain protective barrier between the bond of the wheel and the treatment surface, as a variant - dielectric barrier;

26 Gutsalenko, Yu. G. (2018) Diamond-spark grinding of high functionality materials, $3^{\text {rd }} E d$. Cursor, NTU "KhPI", Kharkov (in Russian).

${ }^{27}$ Agu, A. C., M. D. Uzunyan, and A. V. Rudnev (2018) Grinding of hard alloys by use of minimum quantity lubrication lechnology. NPU «KhPI», Kharkov (In Russian). 
2) use of SLs in the barrier consideration between the wheel bond and the surface of the treatment creates prerequisites for the selective, in the areas of contact sampling of lubricants, and therefore more functionally efficient electro-discharge auto-forming of cutting relief, increases the mode combinatorial capabilities in the management of the cutting relief in DSG process;

3) use of SLs in diamond grinding slows the loss of cutting grain sharpness, and at the same time, with the consequent increase in the life of diamond wheels, contributes to a more pronounced surface roughness;

4) use of SLs in diamond grinding significantly extends the possibilities of controlling the engineering of the surface layer under treatment due to the variation in the composition, intensity and structure of the cycle of SL feed, and at DSG - also in conjunction with the control of the flow of electricity into the cutting zone.

\section{Description of general research structure, purpose and tasks of this work}

The structure of the research is built in motion from virtual computer finite element modeling and analysis of the thermo-physical state of the cutting surface and surface in the processes of diamond-abrasive cutting of difficult-to-process materials using known SLs to a coherently constructed chain of full-scale experiments, in advance, from the very beginning of the project, provided by the original, patent-protected development of a method and device for SL feeding on the cutting surface of the grinding wheel according schemes for continuous and controlled intermittent sliding contact.

The chain of full-scale experiments is based on the tests of known SLs in diamond grinding with thermometric studies in conditions and without electric current in the cutting zone, obtaining and generalization of experimental databases on surface layer X-ray, micrometry and surface microscopy after anhydrous DSG processes, in this case with the use of SLs, everything is done by grinding of hard-to-process materials from a series of hard-alloy and super-hard tools.

Further, as determined from the results of the studies of the physictechnical challenges for improving the SL compositions of diamond grinding of hard-to-process materials, new SL compositions are developed and experimentally tested, with the patenting of selected ones based on the results of tests for practical application. 
Completes the block of experimental research on the project, based on the opportunities provided by the use of SLs, search for new management solutions, first, the development of cutting relief in the DSG processes; secondly, the engineering of the surface layer under diamond-abrasive treatment due to variation in the composition, intensity and structure of the SL feed cycle, and in the case of DSG - also compatible with the control of electrical modes in the cutting zone.

The peculiarities of the individual components of the research are largely determined by the specificity of electro-physical phenomena in the DSG zone in the presence of SL which is considered as a coating with a high wear rate and is renewed according to certain technological regulations.

The aim of this work is to check, demonstrate and comprehend the technological prospects for the use of solid lubricants in the processes of diamond spark grinding of difficult-to-machine materials using the example of tool hard alloy.

The objectives of the research are outlined by the need to reach: first, the technological indicators of DSG with the usual organization of the lubricating and cooling medium (jet watering) and using the technique of minimal lubrication (solid lubricants); second, a comparison of the possibilities of different processing organization; thirdly, the choice of the preferred solid lubricant for further in-depth studies of the diamond spark grinding process with its use; fourth, a comprehensive forecast of the prospects of this processing method with the use of solid lubricants in the direction of increasing technological productivity, functional quality and environmental friendliness of production facilities.

\section{Organization of experimental research}

The process of diamond spark grinding with the use of various solid lubricants was investigated in relation to the processing of T15K6 carbide inserts.

Experimental studies were carried out on the 3D642E model of a universal grinding machine produced at the Mukachevo Machine-Tool Plant in Ukraine and modernized at the NTU "KhPI" to implement the process of diamond spark grinding by installing a power source 6 and additional conductive devices (Fig. 1). A special HO6506 pulse generator, produced on the production base of the Design, Technological and Experimental Institute "Ukrorgstankinprom" in Ukraine (Kharkov), was used as a power source, which converts an alternating current with a 
voltage of $380 \mathrm{~V}$ into a unipolar pulse with an adjustable frequency and current strength.

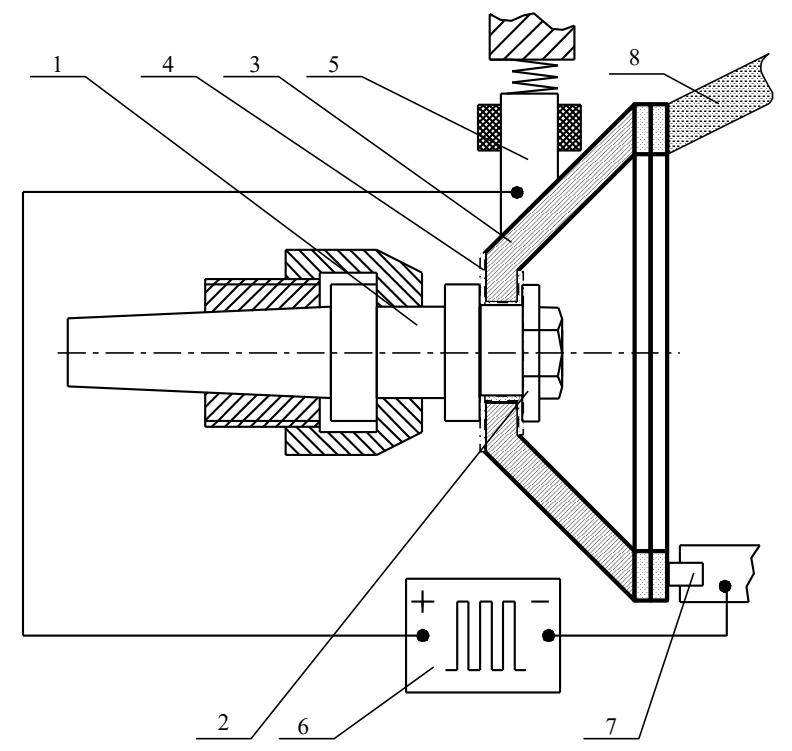

Fig. 1. Upgraded system "machine - device - tool - workpiece" for researching the process of diamond spark grinding:

1 - machine spindle; 2 - details for fastening the wheel; 3 - diamond

wheel wramework; 4 - dielectric coating; 5 - current lead brush; 6 - pulse generator; 7 - processed insert (plate); 8 - solid lubricant

The impulse current was supplied to the grinding zone through the graphite brush 5. At the same time, it was possible to connect one pole of the generator to the brush and through it to the diamond wheel 3, and the other to the processed carbide plate 7 , fixed in the device on the machine table.

The introduction of additional energy into the diamond spark grinding zone with the polarity of the electrodes covering the processing zone (diamond wheel - anode, processed hard-alloy carbide plate - cathode), provides better auto-regulation of the cutting relief of the diamond wheel with a lower energy effect of electric discharges on the processing object (hard alloy), that reduces the likelihood of cracking.

The experiments were carried out with an average value of current $I=4 \ldots 6 \mathrm{~A}$, no-load voltage $U=60 \ldots 80 \mathrm{~V}$, pulse ratio $S=2$ at a pulse frequency $f=66 \mathrm{kHz}$. 
Grinding was carried out at a grinding speed $V=25 \mathrm{~m} / \mathrm{s}$ according to an elastic scheme, when the transverse feed is not rigidly carried out by the feed mechanism, but is set by a certain force using weights on a special device. The normal pressure $P_{\mathrm{n}}$ in the presented series of experiments was $1.2 \mathrm{MPa}$.

In the process of research, cup-shaped diamond wheels 12A2-45 ${ }^{\circ}$ AC6-100 / 80 M1-01-4 were used. For their electrical isolation, a coating 4 with a thickness of $30 \ldots 45 \mu \mathrm{m}$ was applied to the bore hole by the method of plasma electrolytic oxidation according to the previously developed technological recommendations ${ }^{28,29}$.

The solid lubricant was supplied by the contact method. A cast cylinder made of this material with a diameter of $D=10 \ldots 12 \mathrm{~mm}$ was installed in the device and with a small force of $P=5 \mathrm{~g}$ pressed against the wheel at an angle $\alpha=60^{\circ}$, which ensured continuous contact of the lubricant with the working surface of the diamond wheel during grinding.

When choosing the composition of solid lubricants in experimental studies, we were guided by the recommendations ${ }^{30}$, according to which the base components should provide a high lubricating effect - to reduce micro-seizure, scuffing, frictional heating and friction forces in the platetool contact zone. For comparison with the traditional method of jet watering with $3 \%$ soda water solution, three compositions of solid lubricants were used: 1 - technical stearin; 2 - sebacic acid; 3 - a mixture of stearin with sebacic acid (1:1).

\section{Main results and discussion}

The performance of the first task of the work according to the adopted organization of experimental research is summarized in Fig. 2, reflecting information on the minute grinding productivity $\left(Q, \mathrm{~cm}^{3} / \mathrm{min}\right)$, the consumption of superhard abrasive per unit of volumetric material removal $\left(q, \mathrm{mg} / \mathrm{cm}^{3}\right)$ and the technological cost of processing in data conversion to conventional jet watering $(C, \%)$.

${ }^{28}$ Gutsalenko, Yu. G. (2020) In: Modern engineering research: topical problems, challenges and modernity. Baltija Publishing, Riga, 106-126.

29 Sevidova, E., et al. (2020) LNME: Proc. of the 2nd Int. Conf. on Design, Simulation, Manufacturing: The Innovation Exchange, 542-551.

${ }^{30}$ Khudobin, L. V. (Ed.) (2006) Lubricating and cooling technological means and their use in cutting: Handbook. Mechanical Engineering, Moscow (In Russian). 


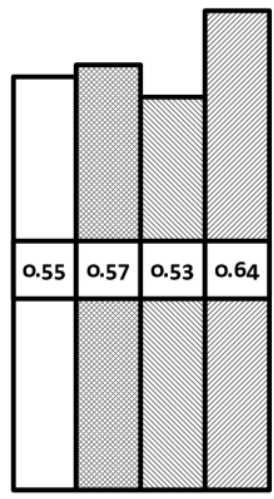

$Q, \mathrm{~cm}^{3} / \min$

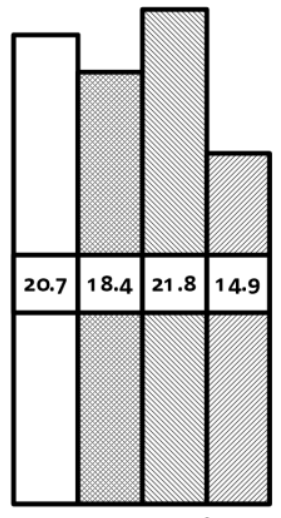

$q, m g / \mathrm{cm}^{3}$

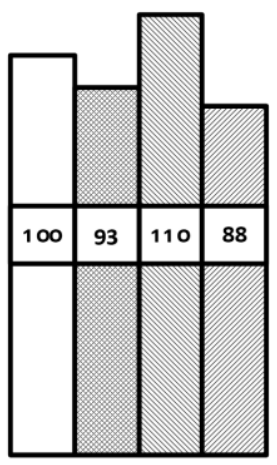

$C, \%$

$\square \quad 3 \%$ soda water solution, jet watering

Stearin, solid lubricant

Sebacic acid, solid lubricant

Mixture of equal parts stearin and sebacic acid, solid lubricant

Fig. 2. Technological parameters of diamond spark grinding of hard alloy T15K6 using lubricating and cooling technological means of different composition and method of serving

The solutions to the second, third and fourth tasks of the work also follow from fig. 2.

Answering the second problem, it is obvious that the organization of treatment with jet watering is superior to the results of the use of sebacic acid, but inferior to the use of stearin and, especially, a mixture of equal parts of stearin and sebacic acid. In the results with the use of acid, an explanation for the increased wear of the tool and, accordingly, a decrease in productivity and an increase in the cost price, apparently should be sought in the excessive renewal of the cutting relief, additionally to the erosion reduction of the wheel metal bond, by means of the acid etching effect as on the inter-granular wheel surface, and on the intra-granular metal binder - the inevitable heredity of the catalytic synthesis of synthetic diamonds ${ }^{31}$.

${ }^{31}$ Novikov, N. V. (Ed.) (1986) Synthetic superhard materials (In 3 Vols), Vol. 1. Naukova Dumka, Kiev (In Russian). 
Answering the third task, the best technological indicators in terms of processing productivity and tool wear, as well as technological cost, in comparison with the usual organization of the lubricating and cooling medium (jet watering with a $3 \%$ soda water solution), and with the independent use of ingredients demonstrates the use of an equilibrium mixtures of technical stearin and sebacic acid. Compared with the traditional support of the process of diamond spark grinding by jet watering using this solid lubricant, the registered increase in processing productivity $(Q)$ is over $15 \%$, and the decrease in tool wear $(q)$ and technological cost $(C)$ is over $25 \%$ and over $10 \%$. respectively. Therefore, we consider the equilibrium mixture of stearin and sebacic acid as promising for further in-depth studies of the diamond spark grinding process with its use.

The presented results of experimental practice allow us to meet the fourth problem. Diamond spark grinding is a promising processing method for the application of minimum lubrication technology. The use of solid lubricants in the working processes of diamond spark grinding can provide a complex effect of increasing both technological productivity (processing productivity) and functional quality (for example, with some modest decrease in the attainable level of processing productivity, by $5-10 \%$ from the obtained data on the use in grinding the hard alloy T5K10 of an equilibrium mixture of stearin and sebacic acid, and thereby providing a "margin of safety" in the formation of favorable thermo-mechanical heredity in the surface layer of the treatment object), while reducing water consumption for production needs and lower technological processing cost.

\section{CONCLUSIONS}

The goal of this work has been achieved - on the experimental basis of the processing of tool hard alloy, technological prospects for the use of solid lubricants in the processes of diamond spark grinding of difficult-to-machine materials have been determined.

Further in-depth studies of the diamond spark grinding process within the framework of the environmentally oriented concept of minimum lubrication are expedient to be carried out using an equilibrium mixture of stearin and sebacic acid, which has proven itself to be effective in supporting the processing of the tool hard alloy T5K10, with an increase in the wear resistance of the tool and the productivity of mass micro- 
cutting with diamond grains of its working surface, while reducing the technological cost.

Thus, further progress in solving the complex problem of technological, qualitative and ecological functionality of the finishing forming for the difficult-to-process materials is associated with using the DSG method with application of SL simultaneously initiating electrodischarge physical effects in the cutting zone in addition to mechanical removal of the allowance.

The technical ideology of the work is based on modern scientific and practical ideas in the fields of engineering, cutting materials and materials science. Scientific novelty follows from the presence of analogues only far from solving the issues of this project.

Work out a system of technical solutions for the method and device for feeding solid lubricants, SL compositions, control of the efficiency of anhydrous diamond grinding processes; scientific and practical guidelines for selection and technological instructions for application of SL as well as management of roughness of the machined surface under anhydrous diamond grinding with SL will be useful results of practical experience, first acquired through scientific research as well as validated, refined, developed.

Waiting for confirmation by thermometric studies - virtual (on finiteelement models) and full-scale (instrument visualization by infrared thermal imager) - for the working hypothesis of a considerable degree of thermal analogy, and possibly the advantage with a lower level of thermal stress of anhydrous DSG processes with SLs compared to the use of conventional grinding with the irrigation scheme for the submission of LCTM is based on the knowledge of tribology and thermal physics of the pronounced discrete (developed, free of shavings relief) and almost continuous (relief in shavings) intense mechanical contacts.

The expectation of the confirmation of microscopic studies of the working hypothesis of the equivalence of anhydrous DSG processes to the processes of diamond grinding with the function of jet cleaning of the wheel working surface and its washing from chips by implementation of the jet scheme of the LCTM submission based on the such known phenomena in the area of cutting at DSG as evaporation of micro-thin chip of sliding contacts in channels of electric micro-discharges between diamond wheel metal bond and surface of machining.

The results of the research on the project with the prospectus provided here, technical solutions, scientific-practical and technological 
recommendations and instructions will significantly develop the theory and practice of grinding.

Resource saving can be considered as a thematic space for further research from the standpoint of reducing the energy consumption of diamond spark grinding with the use of solid lubricants, determining new environmental frontiers and further technological prospects for the development of this method of intensified processing.

The advantage of the expected results is that their use does not require significant financial costs, but on the contrary will save considerable money with obtaining a significant environmental effect without reducing the technological productivity and functional quality of diamond grinding of difficult-to-process materials.

\section{SUMMARY}

The use of solid lubricants in anhydrous grinding technologies is consistent in reducing the use of water to serve industrial needs, which is one of the leading global environmental dominants. It is proposed to carry out development and research of ecological anhydrous processes of abrasive finishing forming of difficult-to-process materials by the method of diamond-spark grinding, which is the founder of NTU "KPI" and which has proved its high innovative flexibility and efficiency by wide scientific and industrial practice. The electric discharge bases of the technological advantages of diamond spark grinding make it possible to consider this processing method among the environmentally promising in nature. A decrease in the thermo-mechanical tension of mass microcutting in the zone of diamond spark grinding, which is ensured by the development of its working relief, supported by the action of electric discharges on the bond of the wheel, also makes it possible to consider approaches to organizing this process using the minimum lubrication methodology. The technologically competitive environment and some hypotheses and bases for the development of a complex of patentable technical solutions, as well as scientific and practical recommendations on the choice and technological instructions for the implementation of solid lubricants in diamond-spark grinding operations are presented. The paper presents and summarizes the experimental results of using a number of solid lubricants, gives practical recommendations and defines the tasks of further research. Work in this direction is focused on reducing the use of water in servicing industrial needs, which is an urgent global environmental challenge to the development of civilization 
and meets the modern technocratic strategies of leading world economies. The presented materials announces the implementation of the project ID: 83995 (3002-6664) under the auspices of the Ministry of Science and Education of Ukraine.

\section{REFERENCES}

1. Kobzar, L. E., V. A. Fadeev, and N. K. Bezzubenko (1995) Progressive diamond-spark grinding. Kharkov State Polytech. Univ., FED Sc.-Prod. Assoc., Kharkov, 153 p. (in Russian).

2. Uzunyan, M. D. (2003) Diamond-spark grinding of hard alloys. NTU "KhPI", Kharkov, 359 p. (in Russian).

3. Gutsalenko, Yu. G. (2018) Diamond-spark grinding of high functionality materials, $3^{\text {rd }}$ Ed. Cursor, NTU “KhPI", Kharkov, 290 p. (in Russian).

4. Gutsalenko, Yu., and C. Iancu (2020) Integrated electric discharge technologies for reliability and durability of mechanical systems in light of modern world civilization challenges. Fiability \& Durability, No. 1(25)/2020, pp. 5-10.

5. Winter, M. (2016) Eco-efficiency of Grinding Processes and Systems. Springer International Publishing AG Switzerland, https://doi.org/10.1007/978-3-319-25205-6.

6. Collins, A., et al. (2019) The Global Risk Report 2019, $14^{\text {th }}$ Ed. World Economic Forum, Geneva, 114 p., http://www3.weforum.org/ docs/WEF_Global_Risks_Report_2019.pdf.

7. Benedicto, E., et al. (2017) Technical, economic and environmental review of the lubrication/cooling Procedia Eng. 184 99-116, DOI 10.1016/j.proeng.2017.04.075.

8. Micron Annual Report 2018 (2018). Micron Holding AG, Biel, https://www.mikron.com/fileadmin/user_upload/01mikron_group/reports /2018/Mikron_AnnualReport2018_WEB.pdf.

9. Smit, J., et al. (2016) Industry 4.0: Study for the European Parliament's Committee on Industry, Research and Energy. EP, Directorate General for Internal Policies, Policy Dept A: Econ. and Sci. Policy, Brussels, $94 \mathrm{p}$.

10. Toward realization of the new economy and society - reform of the economy and society by the deepening of "Society 5.0" (2016). Keidanren (Japan Business Federation), Tokyo, April 19, 2016, 26 p. (Outline), http://www.keidanren.or.jp/en/policy/2016/029_outline.pdf. 
11. Made in China 2025: Global ambitions built on local protection (2017). U.S. Chamber of Commerce, Washington, 84 p., https://www.uschamber.com/sites/default/files/final_made_in_china_202 5_report_full.pdf.

12. Gutsalenko Yu., et al. (2019) Environmental actuality, technological competitive environment and prediction of the prospects of anhydrous diamond-spark grinding using solid lubricants. Annals of the „Constantin Brancusi” University of Targu Jiu, Engineering Series, Iss. 2/2019, pp. 13-17.

13. Yang, M., et al. (2018) Thermodynamic mechanism of nanofluid minimum quantity lubrication cooling grinding and temperature field models. Ch. in: Microfluidics and Nanofluidics. IntechOpen, London, 61-81, DOI 10.5772/intechopen.74969.

14. Hadad, M., and A. Sharbati (2016) Thermal aspects of environmentally friendly-MQL grinding process Procedia CIRP 40 509515, DOI 10.1016/j.procir.2016.01.125.

15. Toyokawa, S., et al. (2019) Surface characteristics with curved grinding of a titanium alloy with coolant supplied from the inner side of the grinding wheel Key Eng. Mater. 825 84-91, DOI 10.4028/www.scientific.net/KEM.825.84.

16. Ohta, Yu., et al. (2017) Efficiency investigation of removal of loading carbon chips on wheel surface using dry ice blasting Key Eng. Mater. 749 124-129, DOI 10.4028/www.scientific.net/KEM.749.124.

17. Boswell, B., et al. (2016) Grinding using cold air cooling Lect. Notes Eng. Comput. Sci. 2 680-687, http://hdl.handle.net/ 20.500.11937/51540.

18. Migranov, M. Sh., et al. (2018) The use of dry electrostatic cooling when cutting of hard materials Bulletin of the UGATU 22 (4(82)): 12-18 (In Russian) http://journal.ugatu.ac.ru/index.php/ Vestnik/article/view/1807.

19. Sen, B., et al. (2019) Eco-friendly cutting fluids in minimum quantity lubrication assisted machining: a review on the perception of sustainable manufacturing Int. J. Pr. Eng. Man.-GT In press, publ. online: 15 Oct. 2019, DOI 10.1007/s40684-019-00158-6.

20. Agu, A. C., and M. D. Uzunyan (2017) Investigation of surface roughness during diamond-spark grinding of hard alloys using minimal lubrication technology Cutting and tooling in technological systems $91 \quad 12-17$ (In Russian), http://library.kpi.kharkov.ua/files/JUR/ rez_87_2017.pdf. 
21. Agu, A. C., M. D. Uzunyan, and A. V. Rudnev (2018) Grinding of hard alloys by use of minimum quantity lubrication lechnology. NTU "KhPI", Kharkov, 140 p. (In Russian), http://repository.kpi.kharkov.ua/ handle/KhPI-Press/38752.

22. Gutsalenko, Yu. G. (2020) Electric insulation technologies and characteristics of micro-arc oxidation of aluminum alloys of diamond grinding wheel wrameworks. Ch. in: Modern engineering research: topical problems, challenges and modernity. Baltija Publishing, Riga, pp. 106-126, https://doi.org/10.30525/978-9934-588-47-1.6.

23. Sevidova, E., et al. (2020) Effect of morphological features on dielectric properties of plasma electrolytic oxidation coatings on D16T aluminum alloy. LNME: Proc. of the 2nd Int. Conf. on Design, Simulation, Manufacturing: The Innovation Exchange, pp. 542-551, https://doi.org/10.1007/978-3-030-50794-7_53.

24. Khudobin, L. V. (Ed.) (2006) Lubricating and cooling technological means and their use in cutting: Handbook. Mechanical Engineering, Moscow, 544 p. (In Russian).

25. Novikov, N. V. (Ed.) (1986) Synthetic superhard materials (In 3 Vols), Vol. 1: Synthesis of superhard materials. Naukova Dumka, Kiev, 264 p. (In Russian).

\section{Information about authors:}

Gutsalenko Yu. G., Senior Staff Scientist, National Technical University "Kharkiv Polytechnic Institute" 2, Kyrpychova str., Kharkiv, 61002, Ukraine

Rudnev A. V., $\mathrm{PhD}$, Senior Staff Scientist, National Technical University "Kharkiv Polytechnic Institute" 2, Kyrpychova str., Kharkiv, 61002, Ukraine 